\title{
General Intelligence and Hypercomputation
}

\author{
Selmer Bringsjord \\ Department of Cognitive Science \\ Department of Computer Science \\ Rensselaer Polytechnic Institute (RPI) \\ Troy NY 12180 USA \\ selmer@rpi.edu
}

From its revolutionary arrival on the behaviorismdominated scene, the information-processing approach to both understanding human intelligence, and to the attempt to bestow such intelligence on a machine, has always proceeded under the assumption that human cognition is fundamentally computation (e.g., see (vE95) in connection with CogSci, and (Hau85) in connection with standard AI). But this approach can no longer leave it at that; it can no longer rest content with the coarse-grained creed that its allegiance is to the view that intelligence consists in processing information. There are two reasons: First, AGI is now on the scene, insisting, rightly, that general intelligence should be the focus. Second, ours is an age wherein the formal science of information processing takes quite seriously a detailed mathematical framework that generates a difficult and profound question: "What kind of information processing should those interested in general intelligence take the mind to rest upon, some form of standard, Turing-level computation, or that and hypercomputation?" My question is not to be confused with: "Which form of Turinglevel computation fits best with human cognition?" This question has been raised, and debated, for decades ${ }^{1}$ A recent position on this question is stated by $\left(\mathrm{LEK}^{+} 06\right)$, who argue that Turing-level neurocomputation, rather than Turing-level quantum computation, should be the preferred type of information processing assumed and used in cognitive science.

Recall that computation is formalized within the space of functions from the natural numbers $N=\{0,1,2, \ldots\}$ (or pairs, triples, quadruples, ... thereof) to the natural numbers; that is, within

$$
\mathcal{F}=\{f \mid f: N \times \ldots \times N \longrightarrow N\} .
$$

This is a rather large set. A very small (but infinite) proper subset of it, $\mathcal{T}$ (hence $\mathcal{T} \subset \mathcal{F}$ ), is composed of functions that Turing machines and their equivalents (Register machines, programs written

Copyright (C) 2009, The Second Conference on Artificial General Intelligence (AGI-09.org). All rights reserved.

${ }^{1}$ E.g., see (Bri91). in modern-day programming languages, the $\lambda$ calculus, etc.; a discussion of these and others in the context of an account of standard computation can be found in Bringsjord (Bri94)) can compute; these are the Turing-computable functions. For example, multiplication is one such function: it's laborious but conceptually trivial to specify a Turing machine that, with any pair of natural numbers $m$ and $n$ positioned on its tape to start, leaves $m \cdot n$ on its tape after its processing is complete. Neurocomputation, as defined by the literature $\left(\mathrm{LEK}^{+} 06\right)$ cite, is Turing-computable computation. If the mind/brain merely neurocomputes, then it can't compute any of the functions in $\mathcal{F}$ that are not in $\mathcal{T}$. (If we let $\mathcal{N}$ denote those functions that neurocomputation can handle, we have that $\mathcal{N}=\mathcal{T}$.) The overwhelming majority of functions in $\mathcal{F}$ would thus be beyond the reach of human persons to compute.

The situation is the same no matter what type of standard computation one selects. For example, those inclined to favor traditional symbolic computation aligned with first-order logic (over, say, connectionism), are at bottom using standard Turing machines. For a more exotic example, consider quantum computers: Standard quantum computers, first introduced by (Deu85), can only compute the functions in $\mathcal{T}$, but as some readers will know, some of this quantum computation can be surprisingly efficient. However, the efficiency of a machine is entirely irrelevant to the class of functions it can compute. All those functions commonly said to be intractable, such as the class of NP-complete functions, are in $\mathcal{T}$. The truly intriguing quantum computers would be those capable of hypercomputation. At the moment, it remains an open question as to whether some recherché forms of quantum computation can compute Turing uncomputable functions. So, where $\mathcal{Q} \subset \mathcal{F}$ contains the functions computable by standard quantum computers, we have $\mathcal{Q}=\mathcal{T}$.

Hypercomputation is the computing, by various extremely powerful machines, of those functions in $\mathcal{F}$ that are beyond the so-called Turing Limit; i.e., 
those functions (composing $\mathcal{H}$ ) in $\mathcal{F}$ that aren't in $\mathcal{T}$. The mathematics of hypercomputation is now quite developed; the machines, definitions, and theorems in question are elegant and informative (e.g., see (SS94, Sie99, EN02, Cop98, HL00; $\mathrm{BKS}^{+} 06$, BZ03)).

The evidence that human persons hypercompute comes in two forms: abstract and empirical ${ }^{2}$ The empirical evidence, in short, consists in the brute fact that the following prophecy of Descartes still stands.

If there were machines which bore a resemblance to our body and imitated our actions as far as it was morally possible to do so, we should always have two very certain tests by which to recognize that, for all that, they were not real men. The first is, that they could never use speech or other signs as we do when placing our thoughts on record for the benefit of others ... And the second difference is, that although machines can perform certain things as well as or perhaps better than any of us can do, they infallibly fall short in others, by which means we may discover that they did not act from knowledge, but only for the disposition of their organs. For while reason is a universal instrument which can serve for all contingencies, these organs have need of some special adaptation for every particular action. From this it follows that it is morally impossible that there should be sufficient diversity in any machine to allow it to act in all the events of life in the same way as our reason causes us to act. ((Des11), p. 116)

The advent of AGI heralds an epoch in which the information-processing approach to intelligence boldly confronts what AI simpliciter has for the most part gradually retreated from: capturing general intelligence. In the face of Descartes' claim, this spells surrender for AI, and an acute challenge for AGI. It's a brute fact that human cognizers, in the logico-mathematical realm, conceive, manipulate, reason over ... the space $\mathcal{H}(\mathcal{F}-\mathcal{T})$ above what Turing machines and their equivalents can muster. Were this not happening, we would not have the mathematics of hypercomputation summarized above, the first part of which was discovered in 1965, when one of the first hypercomputing machines (trial-and-error machines) were specified (Gol65; Put65). In this activity, the humans in question use formal schemes that cannot even be directly represented in any of the languages Turing machines and their equivalents are restricted to using. Many AIniks will doubtless hold that in the future their field will discover how to re-express these highly expressive formal schemes, without loss of meaning, in some standard, austere format used to specify Turing-level computation. But AGI researchers, on the other hand, may choose instead

\footnotetext{
${ }^{2}$ Interested readers can assess some of the abstract evidence presented in $\left(\mathrm{BA}_{0}, \mathrm{BKS}^{+} 06\right)$.
}

to press forward in the hope of devising new formalisms and techniques up to the challenge of the ' $\mathrm{G}$ ' in the acronym for their field.

\section{References}

[BA04] Selmer Bringsjord and Konstantine Arkoudas. The modal argument for hypercomputing minds. Theoretical Computer Science, 317:167-190, 2004.

$\left[\mathrm{BKS}^{+}\right.$06] Selmer Bringsjord, Owen Kellett, Andrew Shilliday, Joshua Taylor, Bram van Heuveln, Yingrui Yang, Jeffrey Baumes, and Kyle Ross. A new Gödelian argument for hypercomputing minds based on the busy beaver problem. Applied Mathematics and Computation, 176:516-530, 2006.

[Bri91] S. Bringsjord. Is the connectionist-logicist clash one of AI's wonderful red herrings? Journal of Experimental \& Theoretical AI, 3.4:319-349, 1991.

[Bri94] S. Bringsjord. Computation, among other things, is beneath us. Minds and Machines, 4.4:469488, 1994

[BZ03] S. Bringsjord and M. Zenzen. Superminds: People Harness Hypercomputation, and More. Kluwer Academic Publishers, Dordrecht, The Netherlands, 2003.

[Cop98] B. J. Copeland. Even Turing machines can compute uncomputable functions. In J. Casti, editor, Unconventional Models of Computation, pages 150164. Springer-Verlag, London, UK, 1998.

[Des11] R. Descartes. The Philosophical Works of Descartes, Volume 1. Translated by Elizabeth S. Haldane and G.R.T. Ross. Cambridge University Press, Cambridge, UK, 1911.

[Deu85] D. Deutsch. Quantum theory, the ChurchTuring principle, and the universal quantum computer. Proceedings of the Royal Society of London, Series A, 400:87-117, 1985.

[EN02] G. Etesi and I. Nemeti. Non-turing computability via malament-hogarth space-times. International Journal of Theoretical Physics, 41(2):341-370, 2002.

[Gol65] M. Gold. Limiting recursion. Journal of Symbolic Logic, 30(1):28-47, 1965.

[Hau85] J. Haugeland. Artificial Intelligence: The Very Idea. MIT Press, Cambridge, MA, 1985.

[HL00] J. D. Hamkins and A. Lewis. Infinite time Turing machines. Journal of Symbolic Logic, 65(2):567$604,2000$.

$\left[\mathrm{LEK}^{+}\right.$06] A. Litt, C. Eliasmith, F. Kroon, S. Weinstein, and P. Thagard. Is the brain a quantum computer? Cognitive Science, 30:593-603, 2006.

[Put65] H. Putnam. Trial and error predicates and a solution to a problem of mostowski. Journal of Symbolic Logic, 30(1):49-57, 1965.

[Sie99] H. T. Siegelmann. Neural Networks and Analog Computation: Beyond the Turing Limit. Birkhäuser, Boston, MA, 1999.

[SS94] H. Siegelmann and E.D. Sontag. Analog computation via neural nets. Theoretical Computer Science, 131:331-360, 1994.

[vE95] Barbara von Eckardt. What is Cognitive Science? MIT Press, Cambridge, MA, 1995. 